\title{
COMPLEXITY OF MATCHING EROSION
}

\author{
THINH D. NGUYEN
}

\begin{abstract}
We restrict the notion of a matching cut to a matching erosion where it is required that one of the two sides of the cut is exactly one side of the matching. Given a graph $G(V, E)$, we shall establish that deciding whether $G$ has a matching erosion is a hard computational problem.
\end{abstract}

\section{BaCKGound And PROBlem STATEMENT}

In the theory of finite undirected graph, a basic notion that is broadly studied is the notion of matching cut. A matching cut in a graph $G$ is defined to be a matching subgraph of $G$ that is also the edge set of a cut. A cut of a graph $G=(V, E)$ is a partition of the vertex set $V$ into two sets $(A, B)$ such that $A$ and $B$ induces two disjoint induced subgraphs that were before incident to some crossing edges which are the edges in the corresponding so-called edge cut set. Specifically, the edge set of the cut $(A, B)$ is then defined to be the subset of $E$ containing all the edges with one endpoint in $A$ and the other endpoint in $B$, i.e. those edges $u v$ with $u \in A$ and $v \in B$. A matching cut is formally defined to be the edge set of such a cut, whenever its edge cut set is indeed a matching. It is worth noting that a matching subgraph which removal increases the number of connected components of a graph $G$ is not necessarily a matching cut, since it may contain edges inside the two separated induced subgraphs. It is a matching cut if it contains exactly the edges of the cut. In order to have more hard computational problems in our still very limited arsenal of proven hard problems, we want to restrict this notion further. We define a matching erosion as a matching cut $(A, B)$ such that $A$ is exactly one side of the matching. It means that each vertex $v \in A$ has exactly one edges out of $A$, and for any two different vertices $u, v \in A$ their crossing edges end in different vertices in $B$. Intuitively, such a matching split $M=(A, B)$ visually "peels" off $A$ from $G$.

Formally, we define a matching erosion of an undirected graph as follows:

Definition 1. Given an undirected graph $G=(V, E)$, a matching erosion $(A, B)$ is a partition of $V$ into two disjoint sets $V=A \cup B$ such that:

- $G[A]$ and $G[B]$ are two disjoint induced subgraphs

- The edge set of the cut is a matching: $M=\{u v \in E \mid u \in A \wedge v \in B\}$ is a matching

- Every $u \in A$ is incident to an edge in $M$

Then, our problem naturally asks whether a given undirected graph $G$ has a matching erosion.

Key words and phrases. cut, graph, matching.

Perebor. 
Definition 2. Matching Erosion of a graph:

Input: An undirected graph $G(V, E)$

Output: YES if $G$ has a matching erosion $(A, B)$, otherwise No

In the next section, we will show that MATCHING ERosion is computationally hard.

We will reduce Exact 3-Set Cover problem to our problem. Exact 3-SeT Cover is another decision problem defined as follows. In EXACT 3-SET Cover, we are given a universe set of elements $U=\left\{e_{1}, e_{2}, \ldots, e_{3 n}\right\}$ and a collection $F=$ $\left\{s_{1}, s_{2}, \ldots, s_{m}\right\}$ of subsets of $U$. Each subset $s_{j}$ in $F$ contains exactly 3 elements $e_{s_{j} 1}, e_{s_{j}}, e_{s_{j} 3} \in U$. The decision problem asks whether there exists a subcollection $F^{\prime} \subseteq F$ such that each element $e_{i}$ of $U$ is contained in exactly one subset $s_{j}$ in $F^{\prime}$. Obviously, $F^{\prime}$ will contain exactly $n$ subsets in the collection $F$. Such a collection $F^{\prime}$ is called an exact cover of $U$.

Definition 3. ExACt 3-Set Cover problem:

Input: a universe $U=\left\{e_{1}, e_{2}, \ldots, e_{3 n}\right\}$ and a collection $F=\left\{s_{1}, s_{2}, \ldots, s_{m}\right\}$ of subsets of $U$, where each $s_{j}$ contains 3 elements $e_{s_{j} 1}, e_{s_{j} 2}, e_{s_{j} 3} \in U$

Output: YES if there exists an exact cover $F^{\prime} \subseteq F$, otherwise No

EXACT 3-Set Cover is shown to be hard by [1]. After describing and proving the correctness of the reduction in the next section, we will establish the following claim.

Claim 4. We have that Exact 3-Set Cover $\leq_{p}$ Matching Erosion

1.1. Known hardness results of matching cut. It is well known that deciding the existence of a matching cut in an undirected graph is NP-hard. The proof of this result can be found in [4]. This is strengthened in [5], where they show that the matching cut problem is hard even when restricted to bipartite graph of diameter 4 , or to general undirected graph of diameter 3 . While the literature on complexity of the matching cut problem are full of beautiful results, we find that it seems very difficult to gain any easy similar construction from those results. In fact, our construction is novel and has its own specific features that are not seen in previous construction for the related problem of matching cut. This is a demonstration for the recurring theme of polynomial-time many-one reductions wherein related decision problems of similar notions require totally different constructions, techniques.

On the positive side, polynomial-time algorithms have been devised and constantly improved for graphs and bipartite graphs of smaller diameter bounds. Interested readers can find efficient algorithms for these special cases of the matching cut problem in $[4,5]$.

1.2. Further research. We can further restrict the notion of a matching erosion to a matching split where the requirement for the set $A$ also applies to $B$. Specifically, every vertex in $A$ and $B$ is required to be incident to exactly one edge in the matching split $M$. Intuitively, a matching split $M=(A, B)$ splits the given graph $G$ into two subgraphs with the same number of vertices, and the edge set of the cut perfectly matches the vertices of the two sides. Formally, we have the following definition

Definition 5. Given an undirected graph $G=(V, E)$, a matching split $(A, B)$ is a partition of $V$ into two disjoint sets $V=A \cup B$ such that: 
- $G[A]$ and $G[B]$ are two disjoint induced subraphs of $G$

- The edge set of the cut is a matching: $M=\{u v \in E \mid u \in A \wedge v \in B\}$ is a matching

- Every $u \in A$ is incident to an edge in $M$

- Every $u \in B$ is incident to an edge in $M$

Then, our problem naturally asks whether a given undirected graph $G$ has a matching split.

Definition 6. Matching Split of a graph:

Input: An undirected graph $G(V, E)$

Output: YES if $G$ has a matching split $(A, B)$, otherwise No

The construction in the next section cannot be easily extended to work for the matching split problem. We leave the conjecture of hardness of this restricted notion of matching split for future research.

CONJECTURE: It is hard to decide whether a graph has a matching split.

\section{Reducing Exact 3-Set Cover to Matching Erosion}

In this section, we prove the claim 4 .

Proof. Describing the construction: Given an instance $(U, F)$ of ExACT 3-SET Cover, we will construct an undirected graph $G=(V, E)$ as the produced instance of our problem Matching Erosion. For each element $e_{i}$ in the universe $U$, we create a new vertex $e_{i} \in V$. Similarly, for each subset $s_{j} \in F$, we create a new $K_{3}$ consisting of 3 new vertices $s_{j, 1}, s_{j, 2}, s_{j, 3} \in V$. For each such subset $s_{j}=$ $\left\{e_{s_{j} 1}, e_{s_{j}}, e_{s_{j} 3}\right\}$, we add the 3 edges to $E$, namely $s_{j, 1} e_{s_{j} 1}, s_{j, 2} e_{s_{j} 2}, s_{j, 3} e_{s_{j} 3}$. Finally, we add all edges between pairs of two different vertices of the $e_{i}$ 's vertices to turn these into a $K_{3 n}$ clique.

Correctness of the construction: Suppose that $(U, F)$ has an exact cover $F^{\prime} \subseteq F$ consistig of $n$ subsets in $F$, then based on that solution to Exact 3-SET Cover, we will easily construct a solution to the produced instance $G(V, E)$ of our problem Matching Erosion. Namely, our matching erosion for $G$ would have $A$ to be the set of all the $K_{3}$ 's of the subsets included in the exact cover. Clearly, each vertex $s_{j, k}$ (where $s_{j}$ is included in the exact cover and $1 \leq k \leq 3$ ) in this set $A$ is connected to exactly one vertex outside of $A$, namely its corresponding element $e_{s_{j} k}$ in the universe $U$. Obviously, this is a matching cut that happens to be also a matching erosion.

Conversely, if $G(V, E)$ has a matching erosion $M=(A, B)$, we shall show that none of the elements $e_{i}$ in the universe is included in $A$. Indeed, if some $e_{i}$ is included in $A$ then at most one $e_{l}$ is not in $A$. This is because if there exist $e_{i} \in A$ and $e_{l 1}, e_{l 2} \notin A$ then $A$ cannot form one side of a matching erosion since $e_{i} \in A$ is incident to two crossing edges $e_{i} e_{l 1}, e_{i} e_{l 2}$, recall that the universe $U$ is turned into a $K_{3 n}$ in $G$. So, if one element in $U$ is included in $A$, at most one element $e_{l}$ can be in $B$, but this is also clearly not the case. Because if so, then all the other element $e_{i}$ 's (which are included in $A$ ) are connected to $e_{l} \in B$ violating the definition of a matching erosion. So, we have shown that if some element $e_{i}$ is included in $A$, then all of the $K_{3 n}$ corresponding to $U$ in $G$ are included in $A$. But, this also cannot be the case for a matching erosion. We need the following observation. 
Observation: For each $K_{3}$ corresponding to a subset $s_{j}$, a vertex $s_{j, k}$ in this $K_{3}$ is included in $A$ iff. all the three vertices of this $K_{3}$ are included in $A$.

Proof. Obviously, by definition of a matching erosion.

Using this observation, we are able to show that a matching erosion cannot include all the $K_{3 n}$ to $A$. Indeed, if so, the matching erosion will then partition the $K_{3}$ 's into two disjoint sets of $K_{3}$ 's, those in $A$ and those in $B$. The $K_{3}$ 's in $B$ would then intuitively form an exact cover for $U$. But, unfortunately, in this case, those vertices of the $K_{3}$ in $A$ cannot have any crossing edge in $M$, thus violating the definition of a matching erosion.

We have therefore shown that none of the elements $e_{i}$ in the universe is included in $A$. This implies that $B$ contains all the $K_{3 n}$. So, the matching erosion needs to partition the set of $K_{3}$ 's into two disjoint sets of $K_{3}$ 's, those in $A$ and those in $B$ like before. But fortunately, the situation is now reversed. the $K_{3}$ 's in $B$ does not need any crossing incident edge (this is only required in a matching split). And, the $K_{3}$ 's in $A$ would form an exact cover for $U$.

\section{Conclusion}

Garey and Johnson [1] shape their theory based on previous works of Cook, Levin and Karp. Johnson [2] moves on with the guide to this theory. As long as we study a mathematical conjecture, we should encourage ourselves to have enough labor hours on classical math books like these. Then, reading some articles on the theory of computing like [3] should be a good practice. Only after that, could we think of the ultimate final for all mathematical sciences.

\section{REFERENCES}

1. Michael R. Garey, David S. Johnson, Computers and Intractability: A Guide to the Theory of NP-Completeness

2. David S. Johnson, The NP-Completeness Column: An Ongoing Guide

3. Phan Dinh Dieu, Le Cong Thanh, Le Tuan Hoa, Average Polyno-mial Time Complexity of Some NP-Complete Problems, Theor. Comput. Sci. 46(3): pp.219-237 (1986)

4. Maurizio Patrignani, Maurizio Pizzonia, The Complexity of the Matching-Cut Problem, Lecture Notes in Computer Science, Jan. 2001

5. Hoang-Oanh Le, Van Bang Le, A complexity dichotomy for Matching Cut in (bipartite) graphs of fixed diameter, arXiv:1804.11102v1

Email address: kosmofarmer@yandex.com 